ECONOMÍA: TEORÍA Y PRÁCTICA • Nueva Época, número 44, enero-junio 2016, pp. 169-192, http://www.izt.uam.mx/economiatyp/ojs

\title{
Coordinación de la política monetaria entre dos bancos centrales: un análisis de teoría de juegos*
}

\section{Monetary Policy Coordination Between Two Central Banks: An Analysis of Game Theory}

Erick José Limas Maldonado**

\section{RESUMEN}

En este trabajo se analizan las condiciones bajo las cuales un país pequeño optaría por seguir un juego tipo Nash o uno de tipo Stackelberg. Para ello, se plantea un modelo de dos economías nacionales, con perturbaciones de oferta y demanda, en el cual el banco central de cada país tiene la tasa de interés como instrumento de política. Se muestra que, ante perturbaciones de la oferta, para el país pequeño es preferible la solución de Stackelberg, mientras que, si las perturbaciones son de la demanda, la solución preferible es la de Nash. Así, el punto clave sería determinar si las perturbaciones están induciendo o no un dilema en la función objetivo del banco central.

Palabras clave: bancos centrales, política monetaria, coordinación, Nash, Stackelberg, teoría de juegos.

Clasificación JEL: E58, F42, C70.

\begin{abstract}
This paper analyzes the conditions under which a small country would opt to follow a Nash-style or a Stackelberg-style game. This poses a two-country model with supply and demand shocks, where interest rate is the central banks' instrument. The article shows that if there are supply shocks, Stackelberg's solution is more suitable for a small country; whereas that in case of demand shocks, the preferred solution is Nash's. Thus, the key point is to determine whether or not perturbations are inducing a trade-off in the central bank's objective function.
\end{abstract}

Keywords: central bancks, monetary policy, coordination, Nash, Stackelberg, game theory.

JEL classification: E58, F42, C70.

\footnotetext{
* Fecha de recepción 25/02/2013. Fecha de aprobación: 29/02/2016.

** Freie Universität Berlin. Correo electrónico: ericklimas@zedat.fu-berlin.de.
} 


\section{INTRODUCCIÓN}

A raíz del creciente grado de apertura de las economías, la adopción de medidas en materia de política monetaria puede afectar y verse afectada tanto por las acciones de política tomadas por otros países, como por perturbaciones exógenas que impactan en el propio. Un fenómeno reciente que puede servir para ilustrar mejor lo anterior es la crisis inmobiliaria acontecida en Estados Unidos (EU), la cual causó que las tasas de interés de importantes economías -por ejemplo, Japón- disminuyeran, con la finalidad de proporcionar liquidez al sistema. Esta interdependencia es en particular relevante para la relación entre México -una economía pequeña- y EU - una grande-, la cual se da en el marco de un área común de comercio no exenta de perturbaciones asimétricas que afectan a ambas naciones.

El principal objetivo de este trabajo es evaluar los beneficios y costos potenciales para un país con una economía pequeña, en términos de bienestar social, de optar por seguir un juego tipo Nash o uno tipo Stackelberg. La pregunta que se pretende responder es la siguiente: ¿Cuál tipo de juego es idóneo para el país pequeño? Para enfrentar la cuestión se plantea un modelo de dos naciones (con una economía reducida y con una mayor, respectivamente), en el que los bancos centrales tienen la tasa de interés real como instrumento de política y donde las perturbaciones pueden ser simétricas o asimétricas.

La hipótesis es que, dependiendo del origen de las perturbaciones que afectan a ambas economías, se pueden obtener mejores resultados para el país pequeño bajo la solución de Stackelberg en algunos casos y, en otros, bajo la solución dada por el equilibrio de Nash. Así, el punto relevante sería determinar si las perturbaciones están induciendo o no un dilema (trade-off) en la función objetivo del banco central. De este modo, no es posible hablar ex ante de la deseabilidad o no de las soluciones propuestas.

El trabajo se compone de cinco apartados. En el primero de ellos se presentan las razones que justifican el uso de la teoría de juegos para tratar el tema de la coordinación de las políticas monetarias, así como una panorámica de los argumentos a favor y en contra de dicha coordinación. En el segundo apartado se presentan y justifican las ecuaciones que conforman el modelo base, el cual considera dos economías, una de ellas relativamente más grande que la otra, y en el que la política monetaria está dirigida por el banco central respectivo.

En el apartado tercero se resuelve el modelo para las distintas situaciones consideradas. Posteriormente, en el cuarto, se realiza un ejercicio numérico para 
analizar los efectos de distintas perturbaciones sobre los diversos equilibrios. En el mismo ejercicio, se muestra una simulación con la finalidad de determinar si los resultados obtenidos son sensibles a cambios en alguno de los coeficientes del modelo. Finalmente, se presentan las conclusiones del presente trabajo.

\section{LA COORDINACIÓN DE LA POLÍTICA MONETARIA}

Uno de los campos con mayor crecimiento dentro del área de la macroeconomía abierta lo constituye el estudio de los problemas de coordinación de políticas monetarias internacionales (Frankel y Rockett, 1988), como un claro reflejo del fuerte grado de interrelación existente entre los distintos países. Prueba de ello es el esfuerzo que distintas naciones han venido emprendiendo en vías de una mayor integración, teniendo como ejemplo destacado la zona del euro.

Esta integración halla una de sus principales causas en el reconocimiento por parte de los actores involucrados de los efectos de desbordamiento (spillover effects) que tienen sus acciones de política sobre el resto de las economías. Dichos efectos tienen saldos tanto positivos como negativos, explicándose de este modo la naturaleza conflictiva inherente a las relaciones entre los países. Un caso particular dentro de la amplia gama de efectos de desbordamiento está dado por la política monetaria que mantiene un país, ya que, por ejemplo, una política monetaria restrictiva puede afectar al tipo de cambio y éste, a su vez, repercutir en la balanza comercial de otro, lo cual podría llevar a que este último reaccionara en aras de contrarrestar los efectos de ello.

Dadas las características conflictivas de las interrelaciones en materia de política monetaria internacional, es que se vuelve pertinente analizarlas a la luz de la teoría de juegos (Goodhart, 1994). Pero, antes, es oportuno señalar que dos de los conceptos de solución más utilizados en la literatura sobre el tema son los equilibrios de Nash y de Stackelberg. El primero se caracteriza por ser una situación tal que, asumiendo un mismo estatus para todos los participantes, ninguno de los agentes tiene incentivos para desviarse del curso que ha seguido (Osborne y Rubinstein, 1994, p. 14).

A diferencia de lo anterior, en la noción del equilibrio de Stackelberg, muy común en la teoría del oligopolio, aparece un componente jerárquico en el sentido de que algunos jugadores, llamados líderes, tienen la capacidad de imponer sobre el resto, conocidos como seguidores, sus propias políticas. Así, el líder elige primero su acción y, una vez informado de ésta, el seguidor elige una acción correspondiente (Fernández, 2002). Sin embargo, la solución de Stackelberg 
suele ser dinámicamente inconsistente debido a que el líder tiene incentivos para mejorar su propia recompensa modificando la acción que había anunciado (Blackburn y Christensen, 1989).

Cabe apuntar, tal como señalan Blackburn y Christensen, que los tipos de juegos referentes a la política monetaria suelen estar considerados dentro de la clase de los juegos de suma no-cero, puesto que la cooperación puede mejorar las recompensas de todos los jugadores, desprendiéndose de lo anterior la conjetura de que posibles comportamientos no cooperativos pueden explicarse a partir de la falta de compromisos obligatorios o debido a fallos de información (Canzoneri y Gray, 1985).

La mayoría de los autores que se han volcado hacia el estudio de la coordinación de la política monetaria internacional lo han hecho con este enfoque, siendo el primero de ellos Koichi Hamada (1976), quien propuso en su trabajo seminal estudiar la interdependencia y naturaleza estratégica de las políticas monetarias a través de un modelo de dos países, los cuales persiguen tanto metas inflacionarias como el equilibrio en la balanza de pagos, teniendo ambos el nivel de crédito interno como único instrumento de política. Dicho investigador muestra que, bajo un régimen de tipo de cambio fijo, la solución no cooperativa es subóptima, ya que dichos objetivos no se pueden alcanzar simultáneamente debido al conflicto de intereses entre los dos países.

La línea abierta por Hamada (1976) encontró terreno fértil, pues se inició una escalada de investigaciones en torno al tema, llegando a su momento más álgido a mediados de los ochenta con un artículo de Rogoff (1985), el cual desató un debate aún no resuelto del todo. La discusión abierta por este autor tuvo tal eco que su trabajo ha sido catalogado por Kempf y Thadden (2008) como uno de los más representativos dentro de la primera generación de modelos que se abocan al análisis de la cooperación en materia monetaria. En el citado texto de Rogoff se desarrolla un modelo de dos países con tipo de cambio flotante, con base en el cual el autor analiza, por un lado, la interacción estratégica entre las respectivas autoridades monetarias de ambas naciones y, por otro, las interacciones estratégicas que involucran tanto a agentes privados como a las autoridades monetarias al interior de un país dado.

El principal resultado encontrado es que, contrario a lo que análisis previos habían establecido, la cooperación creciente en el ámbito de la política monetaria internacional no incrementa automáticamente el bienestar en los países involucrados. De hecho, el bienestar en uno o ambos podría ser más alto si las políticas monetarias se conducen de manera independiente. 
El problema potencial radica en que la cooperación entre los bancos centrales puede exacerbar su falta de credibilidad frente al sector privado. La intuición detrás de lo anterior es que la cooperación monetaria internacional puede incrementar la tasa de inflación de los salarios debido a que los sindicatos se darían cuenta de que cada uno de los bancos centrales tiene incentivos para generar inflación. Sin embargo, señala Rogoff (1985), la cooperación en política monetaria puede ser buena sin ambigüedades sólo si el marco institucional es capaz de eliminar o aminorar este problema de falta de credibilidad de los bancos centrales.

Años después, Carraro y Giavazzi (1988) respondieron al anterior desafío teórico al desarrollar un juego secuencial de dos países con seis agentes: una empresa, un sindicato y un banco central representativos de cada nación. En un principio, se fijan los salarios al interior de cada país y en una siguiente etapa, dados los salarios nominales, toca el turno a los bancos centrales de fijar la oferta monetaria. El modelo muestra que la coordinación de política monetaria puede no ser contraproducente en una situación en la que, ante la presencia de contratos nominales, el banco central tiene el poder de afectar variables reales. A partir del postulado anterior es que los autores demuestran que la cooperación internacional se convierte en la estrategia dominante de los bancos centrales.

Posteriormente, Jensen (1997) llega a resultados similares a los anteriores, pero con la diferencia de que mantiene prácticamente intacto el modelo de Rogoff (1985). Tomando en cuenta que, en ausencia de cooperación, el resultado del juego es socialmente ineficiente (Canzoneri y Gray, 1985), en su trabajo, Jensen rechaza los resultados del de Rogoff, postulando que la afirmación de éste en el sentido de que la cooperación es contraproducente descansa en dos supuestos críticos: el sector privado y la autoridad central están en desacuerdo en cuanto al nivel óptimo de empleo y, por otro lado, al sector privado le da lo mismo enfrentar niveles bajos o altos de inflación. En lo referente al primer supuesto, lo encuentra bastante plausible, justificándolo por la presencia de distorsiones en la economía. Sin embargo, discrepa totalmente del segundo supuesto. ¿Por qué habría -se pregunta este autor- de preocuparse la autoridad central por el nivel de inflación si el sector privado no lo hace? Deduce que lo anterior tiene coherencia sólo si se asume que los agentes del sector privado son unidades "atomísticas"1 cuyas acciones unilaterales no pueden afectar la tasa de inflación.

\footnotetext{
${ }^{1}$ Se utiliza este término para aludir a la incapacidad del agente económico de influir por sí solo en el funcionamiento de la economía. En este sentido, es cercano a la doctrina del atomismo, ya que así como la materia se entiende como la suma de átomos aislados, el sistema económico se entiende como la suma de agentes individuales.
} 
Luego entonces, no importa si el sector privado es modelado como hostil (averse) o no a la inflación. Sin embargo, apunta, si algunos de los agentes del sector privado son tomadores de decisiones no atomísticos, se tiene que el segundo supuesto es difícilmente sostenible. De este modo, Jensen (1997) mantiene en lo esencial el modelo de Rogoff (1985), pero permitiendo ahora la presencia de agentes que tienen la capacidad de fijar salarios y que son no atomísticos y hostiles a la inflación, situación ante la cual la cooperación en materia de política monetaria puede no ser contraproducente, llegando a obtenerse mayores niveles de empleo y menores tasas de inflación. Así, al igual que Canzoneri y Henderson (1991), defiende la existencia de ganancias significativas al coordinar respuestas de cara a perturbaciones macroeconómicas.

Siguiendo una lógica parecida a la planteada por Carraro y Giavazzi (1988), Dixit y Lambertini (1999) realizan una extensión del conocido trabajo de Barro y Gordon (1983), en la que consideran la interacción entre la política monetaria de un banco central común en una unión monetaria y las políticas fiscales de los países miembros de ella. Estos autores encuentran que si todos los agentes están de acuerdo en los niveles ideales de producción y empleo, entonces, la solución óptima puede ser alcanzada a pesar de que no exista consenso sobre los pesos relativos de los dos objetivos (inflación y desempleo) y sin importar quién mueve primero. Hay que notar que, para obtener los resultados anteriores, parten del supuesto de que la coordinación de las políticas monetarias da resultados al menos de la misma magnitud a los de las soluciones de Nash y de Stackelberg.

A diferencia de Jensen (1997), Krolzig (1997) generaliza el modelo de Barro y Gordon (1983) hacia un marco estocástico con la finalidad de refutar la proposición de Rogoff (1985) ya comentada. Partiendo de que los agentes privados poseen información imperfecta acerca de la factibilidad de una posible cooperación monetaria, este investigador encuentra que la cooperación entre los bancos centrales constituye un equilibrio bayesiano perfecto.

Para una panorámica más amplia del debate, es recomendable acudir a Díaz (2005). Su artículo resulta interesante porque abona en las dos direcciones de la discusión: por un lado, especifica situaciones en las que es preferible la solución cooperativa, mientras que, por otro, encuentra escenarios para los cuales las soluciones no cooperativas de Nash y de Stackelberg son superiores. Para mostrar lo anterior, esta autora construye un modelo de dos países estructuralmente idénticos, con las mismas preferencias sobre sus objetivos de inflación y crecimiento, y en el que la oferta monetaria es el único instrumento para hacer 
frente a las perturbaciones que puedan afectar a dichos objetivos. La principal aportación de su trabajo es mostrar que los efectos de las perturbaciones dependen de la estructura económica subyacente, concluyendo que si el instrumento de política es la variación de la oferta monetaria, resultarán inequívocas las ventajas de coordinarse en caso de sufrir una perturbación monetaria. Sin embargo, en caso de enfrentarse a perturbaciones reales, la superioridad de la coordinación dependerá de la elasticidad de la oferta agregada. Si ésta es relativamente inelástica, la coordinación monetaria introduce un sesgo inflacionario, obteniéndose en este caso mejores resultados con las soluciones dadas por Nash y por Stackelberg. Sin dar por concluido el debate, es oportuno recuperar los señalamientos de Fritz y Metzger (2006) en el sentido de que, a pesar de que diversos autores han señalado las ventajas de la cooperación, ésta necesita ser respaldada por un compromiso sincero por parte de cada uno de los agentes y la imposición de una serie de restricciones, en algunos casos críticas, sobre las soberanías nacionales; de ahí que sea muy complicado alcanzar y mantener esa cooperación en materia monetaria. ${ }^{2}$

\section{MODELO TEÓRICO}

En el presente trabajo se considera un modelo de dos países con la finalidad de analizar las interacciones estratégicas en materia de política monetaria entre los bancos centrales respectivos. Cada una de estas economías (una considerada doméstica y la otra externa) está caracterizada tanto por una curva de Phillips como por una curva is (de inversión-ahorro), las cuales pueden ser perturbadas por impactos exógenos. Ambas economías son estructuralmente diferentes en un contexto en el que una de ellas es relativamente más grande que la otra.

Adicionalmente, se considera una condición que vincula el tipo de cambio real entre los dos países con el diferencial de tasas de interés real. Las perturbaciones en sus economías pueden ser simétricas, si son de la misma magnitud, o asimétricas, en caso contrario. Se asume que los bancos centrales tienen metas inflacionarias y de producción, las cuales se verán reflejadas en las funciones objetivo correspondientes.

Siguiendo a Ball (1999), Leitemo (2004) y Leitemo, Roisland y Torvik (2002, 2005), se tiene que el instrumento de política monetaria que utilizarán

\footnotetext{
${ }^{2}$ Una vertiente similar a los juegos aquí planteados está dada por aquellos en los que la autoridad fiscal y el banco central están inmersos en un conflicto de intereses. Ejemplos de estos análisis se pueden encontrar en Leitemo (2004) y en Petit (1989).
} 
ambos bancos centrales será la tasa de interés real. Cabe señalar que, de manera general, el instrumento utilizado por los bancos centrales es la tasa de interés nominal. No obstante lo anterior, si en el corto plazo éstos son capaces de hacer un buen pronóstico de la tasa de inflación, entonces es posible establecer una equivalencia entre las tasas de interés nominal y de interés real (Ball, 1999; Leitemo, 2004; Leitemo, Roisland y Torvik, 2002, 2005). De este modo, la rigidez nominal juega un papel clave, ya que, variando la tasa de interés nominal, la política monetaria estaría variando en la práctica la tasa de interés real (Leitemo, 2004; Clarida, Galí y Gertler, 1999).

El modelo que se utilizará se basa principalmente en los trabajos de Leitemo (2004) y Walsh (2003). La principal diferencia en relación con estos autores es que el primero originalmente analiza un juego al interior de un país entre la autoridad monetaria versus la autoridad fiscal, mientras que en el presente trabajo se propone un juego entre las autoridades monetarias de dos países distintos.

Otro cambio sustancial respecto a Leitemo (2004) es la especificación de las curvas IS, ya que aquí la demanda de un país es función creciente del producto del otro, con la intención de reflejar los efectos de desbordamiento, situación que no es considerada por aquel autor en su trabajo. Esta estructura para el lado de la demanda ya ha sido utilizada en diversos trabajos, entre otros, los de Rogoff (1985), Carraro y Giavazzi (1988), Chang, Muinhos y Teixeira (2002) y Walsh (2003). Las principales variaciones en lo tocante a este último son que él asume dos economías completamente simétricas y que el instrumento de política que utiliza es la tasa de inflación, mientras que en este artículo las economías son distintas y el instrumento de política es la tasa de interés real.

El modelo consta de cinco ecuaciones: la demanda doméstica, la demanda exterior, las respectivas curvas de Phillips y la condición según la cual el tipo de cambio real es determinado por medio de la paridad descubierta de las tasas de interés. Todas las variables se miden en logaritmos, con excepción de la tasa de interés real, $r$, como desviaciones de sus respectivos valores de estado estacionario. Las variables y los parámetros con asterisco corresponden a la economía exterior. Todos los coeficientes son positivos.

Siguiendo a Walsh (2003), la curva de oferta agregada de la economía doméstica está en función del tipo de cambio real, del diferencial entre la inflación observada y la esperada y de una perturbación estocástica. De esta forma, la curva de oferta agregada de la economía doméstica está dada por la siguiente expresión: 


$$
y_{t}=-\omega_{1} q_{t}+\omega_{2}\left(\pi_{t}-E_{t-1}\left[\pi_{t}\right]\right)+\eta_{t} .
$$

Reordenando (1), se puede llegar a la siguiente curva de Phillips con expectativas sobre la inflación:

$$
\pi_{t}=E_{t-1}\left[\pi_{t}\right]+\phi q_{t}+\gamma y_{t}+\varepsilon_{t},
$$

donde $\phi=\omega_{1} / \omega_{2}, \gamma=1 / \omega_{2}, \varepsilon_{t}=-\eta_{t} / \omega_{2}, \pi_{t}=p_{t}-p_{t-1}$ es la inflación; $q_{t} \equiv e_{t}+p_{t}^{*}-p_{t}$ es el tipo de cambio real; $e_{t}$ es el tipo de cambio nominal; $p_{t}^{*}$ y $p_{t}$ son los niveles de precios para la economía exterior y nacional, respectivamente, y $y_{t}$ es la brecha del producto, es decir, la diferencia entre el producto observado y el potencial.

La especificación para la curva de Phillips en (2) se puede considerar como un caso particular, en el sentido de Svensson (1999) y de Ball (1999), de la generalización propuesta por Clarida, Galí y Gertler (1999), con la diferencia de que aquí la inflación no depende, entre otras cosas, de la inflación pasada, sino de la expectativa a partir de la inflación actual. Walsh (2003) deriva el término $E_{t-1}\left[\pi_{t}\right]$ en la ecuación (1) al asumir que el contrato nominal salarial es establecido con un periodo de adelanto, basándose en las expectativas sobre el nivel de precios. ${ }^{3}$

Otra especificación para la curva de Phillips, diferente a la que aquí se plantea y que también es un caso particular de la generalización propuesta en Clarida, Galí y Gertler (1999), consiste en hacer depender la inflación actual de la expectativa sobre la futura. En los trabajos de Leitemo, Roislan y Torvik $(2002,2005)$ se presenta evidencia empírica tanto a favor como en contra de las dos especificaciones de las curvas de Phillips ya mencionadas.

La ecuación (2) es muy similar a una curva de Phillips para una economía cerrada. Sin embargo, dado que el modelo considera economías abiertas, existe una diferencia sustancial entre (2) y la curva de Phillips para economías cerradas, la cual consiste en que (2) incluye un término que refleja el efecto del tipo de cambio real sobre la inflación. Ball (1999), Leitemo (2004) y Chang, Muinhos y Teixeira (2002) derivan la inclusión del tipo de cambio real en (2) al considerar que la inflación en el periodo presente es una media ponderada de la

\footnotetext{
${ }^{3}$ El contrato nominal $w^{c}$ satisface $w_{t}^{c}=E_{t-1}\left[p_{t}\right]+E_{t-1}\left[y_{t}\right]+E_{t-1}\left[n_{t}\right]$, en donde $E_{t-1}$ denota la expectativa condicional sobre la información disponible al final del periodo $t-1 ; p_{t}, y_{t}$ y $n_{t}$ son los logaritmos, en términos de las desviaciones del estado estacionario, del nivel de precios, la producción y la demanda de trabajo, respectivamente (Walsh, 2003, p. 192).
} 
inflación doméstica y la inflación importada, considerando que los precios importados siguen la paridad del poder compra y tomando a $\phi$ como la proporción de los bienes importados.

Así, el parámetro $\phi$ puede ser interpretado desde dos ópticas estrechamente ligadas entre sí: como la fracción de bienes importados en el índice de precios al consumidor (Leitemo, 2004) y como un indicador del grado de apertura de la economía en cuestión (Chang, Muinhos y Teixeira, 2002). Así, si se tuviera que $\phi<\phi^{*}$, se daría el hecho de que la economía doméstica es más cerrada con relación a la otra, debido a que su coeficiente de traspaso del tipo de cambio a la inflación es menor. Dada la definición del tipo de cambio, se tiene que los incrementos de éste implican una depreciación real de la economía doméstica. De manera análoga, la curva de Phillips para la economía exterior está dada por la siguiente expresión:

$$
\pi_{t}^{*}=E_{t-1}\left\lfloor\pi_{t-1}^{*}\right\rfloor-\phi^{*} q_{t}+\gamma^{*} y_{t}^{*}+\varepsilon_{t}^{*} .
$$

Los términos $\varepsilon_{t}$ y $\varepsilon_{t}^{*}$ en (2) y (3) son perturbaciones de la oferta que capturan cualquier fenómeno que podría afectar a la inflación con independencia de los movimientos en la demanda, en la expectativa sobre la inflación o en el tipo de cambio. Estas perturbaciones tienen varianzas $\sigma_{\varepsilon}^{2}$ y $\sigma_{\varepsilon}^{2}$, respectivamente, no están serialmente correlacionadas y su media es cero.

Por otra parte, es necesario especificar una condición para la demanda agregada de la economía doméstica. Esta condición está dada por la siguiente curva Is:

$$
y_{t}=-\alpha r_{t}+\beta q_{t}+\delta y_{t}^{*}+u_{t},
$$

donde $r$ es la tasa de interés real y los parámetros $\delta, \delta^{*}$ indican el tamaño relativo de las economías. De este modo, si se verificase que $\delta>\delta^{*}$, se tendría que la economía doméstica es más dependiente del producto del otro país, indicando con ello que la economía exterior es más grande en relación a la doméstica (Chang, Muinhos y Teixeira, 2002). El signo negativo del coeficiente de la tasa de interés real refleja la sustitución intertemporal de consumo. De este modo, la elasticidad de la tasa interés en la curva is corresponde a la elasticidad intertemporal de sustitución (Clarida, Galí y Gertler, 1999). De manera análoga, la curva Is de la economía exterior está dada por la siguiente expresión:

$$
y_{t}^{*}=-\alpha^{*} r_{t}^{*}-\beta^{*} q_{t}+\delta^{*} y_{t}+u_{t}^{*} .
$$


Los términos $u_{t}$ y $u_{t}^{*}$ en (4) y (5) representan perturbaciones de la demanda con varianzas $\sigma_{u}^{2}$ y $\sigma_{u}^{2}$, respectivamente, no están serialmente correlacionadas y su media es cero. Finalmente, para cerrar el modelo, se tiene la condición correspondiente a la paridad descubierta de tasas de interés: ${ }^{4}$

$$
E_{t}\left[q_{t+1}\right]-q_{t}=r_{t}-r_{t}^{*}
$$

Esta condición supone que los incrementos en la tasa de interés real hacen más atractivos los activos domésticos, implicando de este modo una apreciación en el tipo de cambio, y es muy importante en el modelo, pues captura los efectos de la política monetaria sobre el tipo de cambio. En tanto las estrategias de la política monetaria no induzcan un sesgo sistemático, la naturaleza gaussiana de los impactos IID (independientes e idénticamente distribuidos) es suficiente para garantizar $E_{t-1}\left[\pi_{t}\right]=E_{t-1}\left[\pi_{t}^{*}\right]=0$ (Ellison, Lucio y Vilmunen, 2007; Walsh, 2003).

Con respecto al tipo de cambio real, cuando los impactos son gaussianos y las expectativas son racionales, es posible escribir el tipo de cambio real como $q_{t}=\rho E_{t}\left[q_{t+1}\right]+v_{t}$, donde $0<\rho<1$ y $v_{t}$ es ruido blanco. De lo anterior se sigue que $E_{t}\left[q_{t+1}\right]=0$ en cualquier solución libre de burbujas especulativas, supuesto que se mantendrá a lo largo del presente trabajo (Ellison, Lucio y Vilmunen, 2007; Walsh, 2003; Chang, Muinhos y Teixeira, 2002; Leitemo, 2004). En cuanto al papel de los bancos centrales doméstico y foráneo, se asume que éstos tienen metas inflacionarias y de producción, las cuales se ven reflejadas respectivamente en las siguientes funciones objetivo: ${ }^{5}$

$$
\begin{gathered}
L_{t}=y_{t}^{2}+\lambda \pi_{t}^{2} \\
L_{t}^{*}=\left(y_{t}^{*}\right)^{2}+\lambda^{*}\left(\pi_{t}^{*}\right)^{2} .
\end{gathered}
$$

Estas funciones objetivo reflejan el hecho de que ambos bancos centrales desean minimizar la variabilidad de la inflación y de la producción con respecto a

\footnotetext{
${ }^{4}$ Con está condición, el modelo está completo. De este modo, no es necesario especificar una condición de equilibrio para el mercado de dinero. Lo anterior se debe a que cuando el banco central tiene la tasa de interés como instrumento, ajusta la oferta monetaria para alcanzar la tasa de interés objetivo (Clarida, Galí y Gertler, 1999).

${ }^{5}$ Clarida, Galí y Gertler (1999), citando a Rotemberg y Woodford (1999), y Woodford (1998) justifican este tipo de función de pérdida al señalar que ésta puede ser obtenida a partir de una aproximación cuadrática de una función de utilidad.
} 
sus valores objetivo. Los términos $\lambda>0$ y $\lambda^{*}>0$ indican el peso relativo que cada uno de los bancos centrales le da a cada uno de estos objetivos. Así, si se tiene que $\lambda<1$, se estaría frente a un banco central con mayor preocupación por las desviaciones de la producción respecto a su nivel potencial u objetivo.

A partir de los elementos anteriores, es posible derivar las formas reducidas del modelo. ${ }^{6}$ Para lograr tal propósito, se procede de la siguiente manera: se toma la ecuación (6), recordando que $E\left[q_{t+1}\right]=0$, y se sustituye junto con (5) en la ecuación (4) para obtener:

$$
y_{t}=a_{1} r_{t}+a_{2} r_{t}^{*}+a_{3} u_{t}^{*}+a_{4} u_{t} .
$$

La expresión anterior se sustituye en (5), con lo que se encuentra una forma reducida para $y_{t}^{*}$ :

$$
y_{t}^{*}=a_{1}^{*} r_{t}+a_{2}^{*} r_{t}^{*}+a_{3}^{*} u_{t}^{*}+a_{4}^{*} u_{t} .
$$

Finalmente, para hallar las expresiones reducidas de $\pi_{t}$ y $\pi_{t}^{*}$ combinamos (2) con (9) y (3) con (10), para obtener, respectivamente:

$$
\pi_{t}=E_{t-1}\left[\pi_{t}\right]+b_{1} r_{t}+b_{2} r_{t}^{*}+b_{3} u_{t}^{*}+b_{4} u_{t}+\varepsilon_{t}
$$

y

$$
\pi_{t}^{*}=E_{t-1}\left[\pi_{t}^{*}\right]+b_{1}^{*} r_{t}^{*}+b_{2}^{*} r_{t}+b_{3}^{*} u_{t}+b_{4}^{*} u_{t}^{*}+\varepsilon_{t}^{*} .
$$

Así, se han obtenido las cuatros formas reducidas del modelo -(9) (10) (11) y (12)-, las cuales serán la base, junto con las funciones objetivo (7) y (8), para los juegos que se desarrollarán en las secciones siguientes.

\section{SOLUCIÓN DEL MODELO}

En este apartado se resuelve el modelo para los distintos casos considerados. En primera instancia, se presenta la solución dada por el equilibrio de Nash para, posteriormente, resolver el modelo con un juego a la Stackelberg, en el que el banco central doméstico funge como seguidor y el banco central exterior como el líder.

\footnotetext{
${ }^{6}$ Los coeficientes de las formas reducidas se especifican en el apéndice 1.
} 


\section{Equilibrio de Nash ${ }^{7}$}

Se asume que cada banco central resolverá su problema de optimización tomando como dada la política monetaria del otro banco. Una explicación intuitiva consiste en suponer que ambos bancos centrales eligen sus tasas de interés simultáneamente. En consecuencia, el problema de optimizar la función objetivo del banco central del país doméstico es el siguiente:

$$
\begin{gathered}
\min _{r_{t}} L=y_{t}^{2}+\lambda \pi_{t}^{2} \\
\text { s.a. (9) y (11). }
\end{gathered}
$$

A partir de la condición de primer orden se obtiene la curva de reacción, la cual indica el ajuste sobre la tasa de interés que realizaría el banco central nacional para enfrentar tanto los efectos ocasionados por la política monetaria del país extranjero así como posibles perturbaciones exógenas al sistema:

$$
r_{R}=n_{1} r_{t}^{*}+n_{2} u_{t}^{*}+n_{3} u_{t}+n_{4} \varepsilon_{t} .
$$

De manera análoga, en el caso del banco central exterior, se tiene lo siguiente:

$$
\begin{gathered}
\min _{\substack{r_{*} \\
t}} L^{*}=\left(y_{t}^{*}\right)^{2}+\lambda^{*}\left(\pi_{t}^{*}\right)^{2} \\
\text { s.a. }(10) \mathrm{y}(12),
\end{gathered}
$$

donde la función de reacción para el banco central exterior está dada por

$$
r_{R}^{*}=n_{1}^{*} r_{t}+n_{2}^{*} u_{t}+n_{3}^{*} u_{t}^{*}+n_{4}^{*} \varepsilon_{t}^{*} .
$$

Al resolver el sistema dado por (13) y (14) se llega al par de estrategias $\left(r_{N}, r_{N}^{*}\right)$ que caracterizan el equilibrio de Nash:

$$
r_{N}=N_{1} u_{t}+N_{2} u_{t}^{*}+N_{3} \varepsilon_{t}^{*}+N_{4} \varepsilon_{t}
$$

y

$$
r_{N}^{*}=N_{1}^{*} u_{t}^{*}+N_{2}^{*} u_{t}+N_{3}^{*} \varepsilon_{t}+N_{4}^{*} \varepsilon_{t}^{*}
$$

${ }^{7}$ El valor de los coeficientes de las soluciones de esta sección se especifica en el apéndice 2. 


\section{Equilibrio de Stackelberg ${ }^{8}$}

Aunque esta solución se presenta como no cooperativa, puesto que subyace un espíritu de competencia entre los dos bancos centrales, se puede considerar que se da un primer nivel de coordinación, ya que, al reconocer que un país funge como líder y otro como seguidor, en los hechos estas naciones están ejerciendo una coordinación implícita en el sentido de quién mueve primero y quién después. En el presente apartado se supone que el banco central extranjero se comporta como el líder en el sentido de que elige primero el nivel de su tasa de interés (tomando en cuenta la función de reacción del otro banco) y que, una vez ocurrido lo anterior, el banco central doméstico elige su mejor respuesta. Así, se asume que $\delta>\delta^{*}$, indicando con ello que es la economía pequeña la que sigue a la grande. En consecuencia, el problema del banco central exterior está dado por:

$$
\begin{gathered}
\min _{r_{t}} L^{*}=\left(y_{t}^{*}\right)^{2}+\lambda^{*}\left(\pi_{t}^{*}\right)^{2} \\
\text { s.a. (10) (12) y (13). }
\end{gathered}
$$

De la condición de primer orden, se obtiene la tasa de interés real de Stackelberg para la economía exterior:

$$
r_{s}^{*}=S_{1}^{*} u_{t}^{*}+S_{2}^{*} u_{t}+S_{3}^{*} \varepsilon_{t}+S_{4}^{*} \varepsilon_{t}^{*}
$$

Para encontrar la tasa de interés de Stackelberg para la economía doméstica, basta con llevar (17) a la curva de reacción del país doméstico (13):

$$
r_{s}=S_{1} u_{t}+S_{2} u_{t}^{*}+S_{3} \varepsilon_{t}^{*}+S_{4} \varepsilon_{t}
$$

Así, (17) y (18) constituyen la solución de Stackelberg.

\section{EJERCICIO NUMÉRICO}

En este apartado se exponen una serie de simulaciones con la finalidad de analizar los efectos de distintas perturbaciones sobre los diversos equilibrios. Es de recordar que, en el caso de la economía doméstica, los coeficientes $\phi$ y $\gamma$ indican el efecto del tipo de cambio real y de la demanda sobre la inflación, respecti-

\footnotetext{
${ }^{8}$ El valor de los coeficientes de las soluciones de esta sección se especifica en el apéndice 3.
} 
vamente; $\alpha$ refleja la elasticidad de la tasa de interés real en la curva IS, $\beta$ indica el efecto del tipo de cambio real sobre la demanda, mientras que $\delta$ mide el grado de dependencia de la economía doméstica respecto a la extranjera. Finalmente, $\lambda$ indica el peso asignado por el banco central al objetivo inflacionario. De manera análoga, los coeficientes con asterisco corresponden a los mismos parámetros de la economía extranjera.

Para el caso de la economía doméstica, los valores de $\phi=0.31$ y $\alpha=0.39$ provienen de Freitas y Muinhos (2001); $\gamma=0.1$ y $\beta=0.08$, de Bonomo y Brito (2001); $\delta=0.2$ y $\lambda=1$, de Chang, Muinhos y Teixeira (2002). Para el caso de la economía exterior, los valores de $\phi^{*}=0.4, \gamma^{*}=0.2, \quad \alpha^{*}=0.6$ y $\beta^{*}=0.2$ se tomaron de Ball (1999), mientras que $\lambda^{*}=1$ y $\delta^{*}=0.1$ provienen de Chang, Muinhos y Teixeira (2002).

En el cuadro 1 aparecen los valores asignados tanto a los coeficientes como a las perturbaciones, mientras que en el cuadro 2 se muestran los resultados que se obtuvieron para las tasas de interés y las funciones de pérdida a partir de dichos valores. Una vez asignados los valores a los coeficientes del modelo, se realizan cinco simulaciones.

Cuadro 1. Valores de los parámetros

\begin{tabular}{lllllllllllllllllll}
\hline & $\lambda$ & $\lambda^{*}$ & $\varepsilon_{t}$ & $u_{t}$ & $\varepsilon_{t}^{*}$ & $u_{t}^{*}$ & $\phi$ & $\gamma$ & $\alpha$ & $\beta$ & $\delta$ & $\phi^{*}$ & $\gamma^{*}$ & $\alpha *$ & $\beta^{*}$ & $\delta^{*}$ \\
\hline $\begin{array}{l}\text { Perturbaciones simétricas } \\
\text { de la demanda }\end{array}$ & 1 & 1 & 0 & 1 & 0 & 1 & 0.31 & 0.1 & 0.39 & 0.08 & 0.2 & 0.4 & 0.2 & 0.6 & 0.2 & 0.1 \\
$\begin{array}{l}\text { Perturbaciones asimétricas } \\
\text { de la demanda }\end{array}$ & 1 & 1 & 0 & 1 & 0 & 2 & 0.31 & 0.1 & 0.39 & 0.08 & 0.2 & 0.4 & 0.2 & 0.6 & 0.2 & 0.1 \\
$\begin{array}{l}\text { Perturbaciones simétricas } \\
\text { de la oferta }\end{array}$ & 1 & 1 & 1 & 0 & 1 & 0 & 0.31 & 0.1 & 0.39 & 0.08 & 0.2 & 0.4 & 0.2 & 0.6 & 0.2 & 0.1 \\
$\begin{array}{l}\text { Perturbaciones asimétricas } \\
\text { de la oferta }\end{array}$ & 1 & 1 & 1 & 0 & 2 & 0 & 0.31 & 0.1 & 0.39 & 0.08 & 0.2 & 0.4 & 0.2 & 0.6 & 0.2 & 0.1 \\
\hline
\end{tabular}

En el primer caso se presenta un entorno en el que no ocurren perturbaciones de la oferta pero sí de la demanda, las cuales se asumen simétricas. De igual forma, en el segundo ejercicio se supone que no existen perturbaciones de la oferta pero sí de la demanda, las cuales se presume son asimétricas. En la tercera simulación se muestra una situación en la cual no existen perturbaciones de la demanda pero sí perturbaciones simétricas de la oferta. En el cuarto ejercicio se analiza un escenario en el que las únicas perturbaciones son asimétricas y de 
Cuadro 2. Resultados de las simulaciones

\begin{tabular}{llcccc}
\hline & & $r$ & $r^{*}$ & $L$ & $L^{*}$ \\
\hline Perturbaciones simétricas & Nash & 2257 & 1925 & 0.0150 & 0.0202 \\
de la demanda & Stackelberg & 2255 & 1915 & 0.0157 & 0.0201 \\
Perturbaciones asimétricas & Nash & 2827 & 3112 & 0.0110 & 0.01482 \\
de la demanda & Stackelberg & 2829 & 3121 & 0.0115 & 0.01476 \\
$\begin{array}{l}\text { Perturbaciones simétricas } \\
\text { de la oferta }\end{array}$ & Nash & 1097 & 11930 & 13954 \\
$\begin{array}{l}\text { Perturbaciones asimétricas } \\
\text { de la oferta }\end{array}$ & Stackelberg & 1340 & 1011 & 11399 & 13893 \\
& Nash & 1500 & 1730 & 16220 & 41614 \\
\hline
\end{tabular}

la oferta. Finalmente, en el último ejercicio se realiza una simulación para distintos valores de $\delta$, manteniendo constantes el resto de los coeficientes. ${ }^{9}$

Los resultados muestran un hecho interesante: en el caso de las perturbaciones de la demanda, ya sea simétricas o asimétricas, se tiene que la solución de Nash para el país doméstico es superior, en el sentido de Pareto, a la de Stackelberg. La intuición señala el hecho de que las perturbaciones de la demanda presionan tanto a la inflación como a la producción en el mismo sentido, por lo que cada banco central no enfrenta en el corto plazo un dilema entre inflación y producción. Así, si se diera una caída en la demanda, esto provocaría un menor nivel de inflación y de producción y, en ese caso, una política adecuada podría ser el mantener una tasa de interés baja para estabilizar tanto la inflación como la demanda (Leitemo, Roisland y Torvik, 2002; Clarida, Galí y Gertler, 1999). De esta forma, al no existir un dilema, el banco central doméstico puede atacar al mismo tiempo los dos objetivos de su respectiva función de pérdida; de ahí que un juego tipo Stackelberg produzca un menor bienestar.

Sin embargo, en el caso de la economía exterior se obtiene un resultado superior, en el sentido de Pareto, con un juego tipo Stackelberg que con uno a la Nash. La intuición de lo anterior radica en que, si bien el líder tampoco enfrenta un dilema entre inflación y producción, sí puede sacar provecho de su posición, al mover primero, para obtener alguna ventaja a costa del país seguidor.

\footnotetext{
${ }^{9}$ Las perturbaciones asimétricas mostradas en el ejercicio corresponden a la circunstancia en que éstas son mayores en la economía exterior que en la economía doméstica. El caso opuesto no se muestra porque, como se verá más adelante, la lógica es similar: el punto relevante es determinar si las perturbaciones, sin importar su signo, inducen un dilema en la función objetivo del banco central.
} 
A diferencia de lo ocurrido con las perturbaciones de la demanda, se tiene que cuando son de la oferta, sí se genera un dilema para el banco central (Leitemo, Roisland y Torvik, 2002; Clarida, Galí y Gertler, 1999). La idea detrás de lo anterior se halla en que, si un impacto en la oferta presiona la inflación al alza, al mismo tiempo estaría reduciendo la producción. Así, una política destinada a disminuir la inflación provocaría una caída mayor en la producción y el empleo. En este caso, el banco central no podría atacar por sí solo y simultáneamente los dos objetivos de su función de pérdida, con lo cual resultaría conveniente para ambos bancos ser partícipes de un juego a la Stackelberg, ya que, al entrar en una dinámica líder-seguidor, se estaría colaborando para atacar ese dilema.

Otro punto importante a considerar es si los resultados son sensibles ante cambios en los coeficientes del modelo. En la gráfica 1 se muestra el comportamiento de la función objetivo del banco central doméstico para los equilibrios de Nash y de Stackelberg con distintos valores de $\delta$ en una situación en la que las perturbaciones son simétricas de la demanda.

Gráfica 1. Comportamiento de la función objetivo del banco central doméstico (L) para los equilibrios de Nash y de Stackelberg con distintos valores de $\delta$

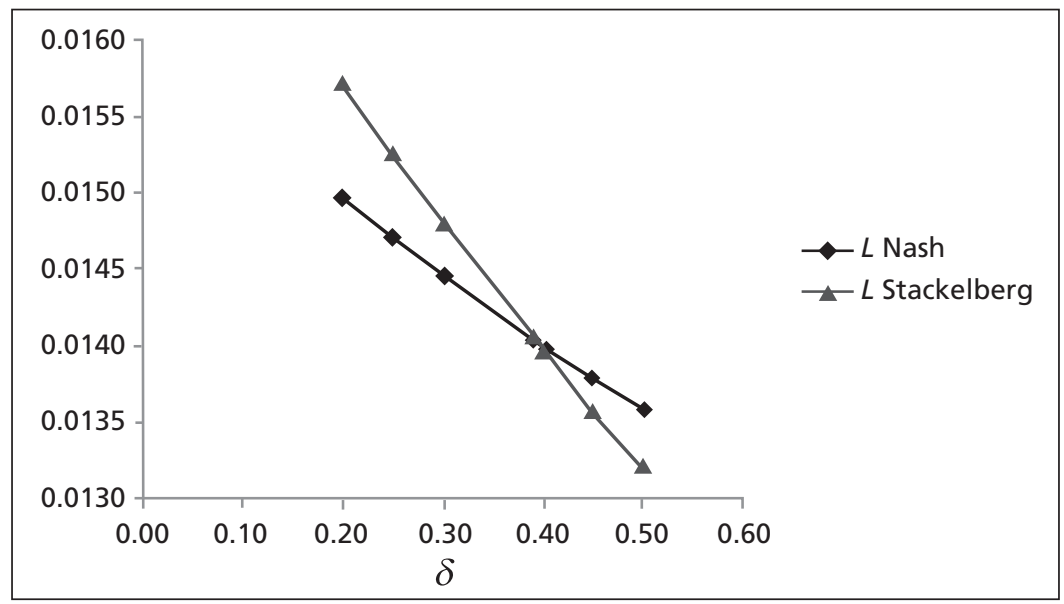

En las simulaciones con perturbaciones de la demanda se mostró que el equilibrio de Nash es preferible sobre el de Stackelberg para el caso de la economía doméstica. Sin embargo, en la simulación mostrada en la gráfica 1 es posible ver que, a partir de $\delta=0.4$, la solución dada por el equilibrio de Stackelberg es preferible a la de Nash debido a que logra minimizar en mayor cuantía la función 
objetivo del banco central doméstico. La intuición de lo anterior radica en el hecho de que a medida que se incrementa el valor de $\delta$, la economía doméstica es cada vez más dependiente de la extranjera, por lo que le resulta conveniente entrar en una dinámica líder-seguidor. ${ }^{10}$

\section{CONCLUSIONES}

En el presente trabajo se modeló un escenario con las economías de dos países estructuralmente diferentes para analizar las interacciones estratégicas en materia de política monetaria entre sus bancos centrales. Cada economía fue caracterizada tanto por una curva de Phillips como por una curva is. En lo referente a los bancos centrales de cada país, se asumió que éstos tienen metas inflacionarias y de producción y que su instrumento de política monetaria es la tasa de interés real.

En el caso de la economía doméstica, se encontró que, ante perturbaciones de la demanda simétricas y asimétricas, la solución dada por el equilibrio de Nash es preferible a la de Stackelberg. La explicación estriba en el hecho de que estas perturbaciones no generan un dilema entre producción e inflación en la función de pérdida del banco central doméstico. Así, ante cualquier impacto en la demanda, el banco central doméstico estaría en la posibilidad de estabilizar por sí solo ambos objetivos al mismo tiempo, por lo que no tendría incentivos para tomar el papel de seguidor en un juego a la Stackelberg. Sin embargo, se mostró que a medida que se incrementa el valor de $\delta$, existe un punto de cruce a partir del cual es preferible que el banco central doméstico ingrese en un juego a la Stackelberg. Esto, debido a que mayor $\delta$ implica que la economía doméstica sea cada vez más dependiente de la extranjera y que, por ello, le resulte conveniente entrar en una dinámica líder-seguidor.

Cuando las perturbaciones son de la oferta, la situación toma un matiz distinto. En ese caso, el banco central doméstico sí tiene incentivos para entrar en un juego a la Stackelberg, debido a que estas perturbaciones generan un dilema entre inflación y producción en su función de pérdida. De tal suerte que actuando de manera unilateral -es decir, con un juego a la Nash- no podría atacar ambos objetivos al mismo tiempo. Pero en un juego a la Stackelberg, el banco central doméstico estaría estableciendo una coordinación implícita al asumir su

\footnotetext{
${ }^{10}$ También se realizaron simulaciones para distintos valores de $\phi, \phi^{*}$ y $\delta^{*}$. En el caso de $\phi$, no se encontraron cambios significativos para la economía doméstica, en tanto que para distintos valores de $\phi^{*}$ y $\delta^{*}$, se mantuvo la superioridad de la solución de Stackelberg para la economía extranjera.
} 
papel de seguidor y actuar en función de lo hecho por el líder. De este modo, aminoraría el dilema en su función objetivo. Estos hallazgos son consistentes con los expuestos por sugeridos por Leitemo, Roisland y Torvik (2002) y Clarida, Galí y Gertler (1999), para quienes un elemento de gran pertinencia es identificar si las perturbaciones, en este caso de la oferta, inducen un dilema para el banco central.

En el caso del banco central de la economía exterior, se tiene que, sin importar el origen de las perturbaciones, la solución dada por el equilibrio de Stackelberg es superior a la dada por el Nash. La intuición detrás de este resultado radica en que, para todos los casos considerados, el banco central exterior funge como el líder. Es precisamente esta ventaja de mover primero la que le otorga al líder una ganancia adicional que no sería factible bajo una situación tipo Nash. Lo anterior refleja que, tal y como se planteó al principio de este trabajo, lo deseable que resulte una $\mathrm{u}$ otra de las soluciones depende del tipo de impactos que enfrenta el banco central. Es decir, no se puede establecer a priori la superioridad de cualquiera de ellas.

Finalmente, cabe señalar que en el presente trabajo sólo se consideraron las interacciones entre los dos bancos centrales. Una extensión interesante sería introducir una autoridad fiscal en cada uno de los países del modelo para, de este modo, añadir a los juegos entre ambos bancos interacciones entre la política monetaria y la fiscal.

\section{ApÉNDiCe 1. COEFICIENTES de la FORMA REDUCIDA DEL MOdElo}

$$
\begin{array}{ll}
a_{1}=\frac{\delta \beta^{*}-\alpha-\beta}{1-\delta \delta^{*}} & a_{2}=\frac{\beta-\delta \alpha^{*}-\delta \beta^{*}}{1-\delta \delta^{*}} \\
a_{3}=\frac{\delta}{1-\delta \delta^{*}} & a_{4}=\frac{1}{1-\delta \delta^{*}} \\
a_{1}^{*}=\frac{\delta^{*} \beta-\alpha^{*}-\beta^{*}}{1-\delta \delta^{*}} & a_{2}^{*}=\frac{\beta^{*}-\delta^{*} \alpha-\delta^{*} \beta}{1-\delta \delta^{*}} \\
a_{3}^{*}=\frac{\delta^{*}}{1-\delta \delta^{*}} & a_{4}^{*}=\frac{1}{1-\delta \delta^{*}} \\
b_{1}=\gamma \frac{\delta \beta^{*}-\alpha-\beta}{1-\delta \delta^{*}}-\phi & b_{2}=\gamma \frac{\beta-\delta \alpha^{*}-\delta \beta^{*}}{1-\delta \delta^{*}}+\phi
\end{array}
$$


188 ECONOMÍA: TEORÍA Y PRÁCTICA • Nueva Época, número 44, enero-junio 2016

$$
\begin{array}{ll}
b_{3}=\gamma \frac{\delta}{1-\delta \delta^{*}} & b_{4}=\gamma \frac{1}{1-\delta \delta^{*}} \\
b_{1}^{*}=\gamma^{*} \frac{\delta^{*} \beta-\alpha^{*}-\beta^{*}}{1-\delta \delta^{*}}-\phi^{*} & b_{2}^{*}=\gamma^{*} \frac{\beta^{*}-\delta^{*} \alpha-\delta^{*} \beta}{1-\delta \delta^{*}}+\phi^{*} \\
b_{3}^{*}=\gamma^{*} \frac{\delta^{*}}{1-\delta \delta^{*}} & b_{4}^{*}=\gamma^{*} \frac{1}{1-\delta \delta^{*}}
\end{array}
$$

\section{Apéndice 2. COEFICIENTES de La SOluCión de NASH}

$$
\begin{aligned}
& n_{1}=\frac{\left(\frac{\delta \beta^{*}-\alpha-\beta}{1-\delta \delta^{*}}\right)\left(\frac{\beta-\delta \alpha^{*}-\delta \beta^{*}}{1-\delta \delta^{*}}\right)+\lambda\left(\gamma \frac{\delta \beta^{*}-\alpha-\beta}{1-\delta \delta^{*}}-\phi\right)\left(\gamma \frac{\beta-\delta \alpha^{*}-\delta \beta^{*}}{1-\delta \delta^{*}}+\phi\right)}{-\left(\left(\frac{\delta \beta^{*}-\alpha-\beta}{1-\delta \delta^{*}}\right)^{2}+\lambda\left(\gamma \frac{\delta \beta^{*}-\alpha-\beta}{1-\delta \delta^{*}}-\phi\right)^{2}\right)} \\
& n_{2}=\frac{\left(\frac{\delta \beta^{*}-\alpha-\beta}{1-\delta \delta^{*}}\right)\left(\frac{\delta}{1-\delta \delta^{*}}\right)+\lambda\left(\gamma \frac{\delta \beta^{*}-\alpha-\beta}{1-\delta \delta^{*}}-\phi\right)\left(\frac{\gamma \delta}{1-\delta \delta^{*}}\right)}{-\left(\left(\frac{\delta \beta^{*}-\alpha-\beta}{1-\delta \delta^{*}}\right)^{2}+\lambda\left(\gamma \frac{\delta \beta^{*}-\alpha-\beta}{1-\delta \delta^{*}}-\phi\right)^{2}\right)} \\
& n_{3}=\frac{\left(\frac{\delta \beta^{*}-\alpha-\beta}{1-\delta \delta^{*}}\right)\left(\frac{1}{1-\delta \delta^{*}}\right)+\lambda\left(\gamma \frac{\delta \beta^{*}-\alpha-\beta}{1-\delta \delta^{*}}-\phi\right)\left(\frac{\gamma}{1-\delta \delta^{*}}\right)}{-\left(\left(\frac{\delta \beta^{*}-\alpha-\beta}{1-\delta \delta^{*}}\right)^{2}+\lambda\left(\gamma \frac{\delta \beta^{*}-\alpha-\beta}{1-\delta \delta^{*}}-\phi\right)^{2}\right)} \\
& n_{4}=\frac{\lambda\left(\gamma \frac{\delta \beta^{*}-\alpha-\beta}{1-\delta \delta^{*}}-\phi\right)}{-\left(\left(\frac{\delta \beta^{*}-\alpha-\beta}{1-\delta \delta^{*}}\right)^{2}+\lambda\left(\gamma \frac{\delta \beta^{*}-\alpha-\beta}{1-\delta \delta^{*}}-\phi\right)^{2}\right)} \\
& n_{1}^{*}=\frac{\left(\frac{\delta^{*} \beta-\alpha^{*}-\beta^{*}}{1-\delta \delta^{*}}\right)\left(\frac{\beta^{*}-\delta^{*} \alpha-\delta^{*} \beta}{1-\delta \delta^{*}}\right)+\lambda^{*}\left(\gamma^{*} \frac{\delta^{*} \beta-\alpha^{*}-\beta^{*}}{1-\delta \delta^{*}}-\phi^{*}\right)\left(\gamma^{*} \frac{\beta^{*}-\delta^{*} \alpha-\delta^{*} \beta}{1-\delta \delta^{*}}+\phi^{*}\right)}{-\left[\left(\frac{\delta^{*} \beta-\alpha^{*}-\beta^{*}}{1-\delta \delta^{*}}\right)^{2}+\lambda^{*}\left(\gamma^{*} \frac{\delta^{*} \beta-\alpha^{*}-\beta^{*}}{1-\delta \delta^{*}}-\phi^{*}\right)^{2}\right]}
\end{aligned}
$$




$$
\begin{aligned}
& n_{2}^{*}=\frac{\left(\frac{\delta^{*} \beta-\alpha^{*}-\beta^{*}}{1-\delta \delta^{*}}\right)\left(\frac{\delta^{*}}{1-\delta \delta^{*}}\right)+\lambda^{*}\left(\gamma^{*} \frac{\delta^{*} \beta-\alpha^{*}-\beta^{*}}{1-\delta \delta^{*}}-\phi^{*}\right)\left(\frac{\gamma^{*} \delta^{*}}{1-\delta \delta^{*}}\right)}{-\left[\left(\frac{\delta^{*} \beta-\alpha^{*}-\beta^{*}}{1-\delta \delta^{*}}\right)^{2}+\lambda^{*}\left(\gamma^{*} \frac{\delta^{*} \beta-\alpha^{*}-\beta^{*}}{1-\delta \delta^{*}}-\phi^{*}\right)^{2}\right]} \\
& n_{3}^{*}=\frac{\left(\frac{\delta^{*} \beta-\alpha^{*}-\beta^{*}}{1-\delta \delta^{*}}\right)\left(\frac{1}{1-\delta \delta^{*}}\right)+\lambda^{*}\left(\gamma^{*} \frac{\delta^{*} \beta-\alpha^{*}-\beta^{*}}{1-\delta \delta^{*}}-\phi^{*}\right)\left(\frac{\gamma^{*}}{1-\delta \delta^{*}}\right)}{-\left[\left(\frac{\delta^{*} \beta-\alpha^{*}-\beta^{*}}{1-\delta \delta^{*}}\right)^{2}+\lambda^{*}\left(\gamma^{*} \frac{\delta^{*} \beta-\alpha^{*}-\beta^{*}}{1-\delta \delta^{*}}-\phi^{*}\right)^{2}\right]} \\
& n_{4}^{*}=\frac{\lambda^{*}\left(\gamma^{*} \frac{\delta^{*} \beta-\alpha^{*}-\beta^{*}}{1-\delta \delta^{*}}-\phi^{*}\right)}{-\left[\left(\frac{\delta^{*} \beta-\alpha^{*}-\beta^{*}}{1-\delta \delta^{*}}\right)^{2}+\lambda^{*}\left(\gamma^{*} \frac{\delta^{*} \beta-\alpha^{*}-\beta^{*}}{1-\delta \delta^{*}}-\phi^{*}\right)^{2}\right]} \\
& N_{1}=\frac{n_{1} n_{2}^{*}+n_{3}}{1-n_{1} n_{1}^{*}} \quad N_{2}=\frac{n_{1} n_{3}^{*}+n_{2}}{1-n_{1} n_{1}^{*}} \\
& N_{3}=\frac{n_{1} n_{4}^{*}}{1-n_{1} n_{1}^{*}} \quad N_{4}=\frac{n_{4}}{1-n_{1} n_{1}^{*}} \\
& N_{1}^{*}=\frac{n_{1}^{*} n_{2}+n_{3}^{*}}{1-n_{1} n_{1}^{*}} \quad N_{2}^{*}=\frac{n_{1}^{*} n_{3}+n_{2}^{*}}{1-n_{1} n_{1}^{*}} \\
& N_{3}^{*}=\frac{n_{1}^{*} n_{4}}{1-n_{1} n_{1}^{*}} \quad N_{4}^{*}=\frac{n_{4}^{*}}{1-n_{1} n_{1}^{*}}
\end{aligned}
$$

\section{Apéndice 3. Coeficientes de la SOlución de Stackelberg}

$$
\begin{array}{ll}
S_{1}^{*}=\frac{f_{1} f_{3}+\lambda^{*} g_{1} g_{3}}{-\left(f_{1}^{2}+\lambda^{*} g_{1}^{2}\right)} & S_{2}^{*}=\frac{f_{1} f_{2}+\lambda^{*} g_{1} g_{2}}{-\left(f_{1}^{2}+\lambda^{*} g_{1}^{2}\right)} \\
S_{3}^{*}=\frac{f_{1} f_{4}+\lambda^{*} g_{1} g_{4}}{-\left(f_{1}^{2}+\lambda^{*} g_{1}^{2}\right)} & S_{4}^{*}=\frac{\lambda^{*} g_{1}}{-\left(f_{1}^{2}+\lambda^{*} g_{1}^{2}\right)} \\
S_{1}=n_{1} S_{2}^{*}+n_{3} & S_{2}=n_{1} S_{1}^{*}+n_{2} \\
S_{3}=n_{1} S_{4}^{*} & S_{4}=n_{1} S_{3}^{*}+n_{4} \\
f_{1}=a_{1}^{*}+a_{2}^{*} n_{1} & f_{2}=a_{2}^{*} n_{3}+a_{3}^{*}
\end{array}
$$




$$
\begin{array}{ll}
f_{3}=a_{2}^{*} n_{2}+a_{4}^{*} & f_{4}=a_{2}^{*} n_{4} \\
g_{1}=b_{1}^{*}+b_{2}^{*} n_{1} & g_{2}=b_{2}^{*} n_{3}+b_{3}^{*} \\
g_{3}=b_{2}^{*} n_{2}+b_{4}^{*} & g_{4}=b_{2}^{*} n_{4}
\end{array}
$$

\section{REFERENCIAS BIBLIOGRÁFICAS}

Ball, Laurence (1999), "Policy rules for open economies", en John B. Taylor (ed.), Monetary Policy Rules, Chicago, The University of Chicago Press, pp. 127-144.

Barro, Robert J., y Gordon, B. David (1983), "Rules, discretion and reputation in a model of monetary policy", Journal of Monetary Economics, 12 (1), pp. 1001-121.

Blackburn, Keith, y Christensen, Michael (1989), "Monetary policy and policy credibility: theories and evidence", Journal of Economic Literature, 27 (1), pp. 1-45.

Bonomo, Marco Antonio, y Brito, Ricardo R. (2001), "Regras Monetárias e Dinâmica Macroeconômica no Brasil: Uma Abordagem de Expectativas Racionais", Working Paper 28, Banco Central do Brasil,.

Canzoneri, Matthew, y Gray, Jo Anna (1985), "Monetary policy games and the consequences of non-cooperative behavior", International Economic Review, 26 (3), pp. 547-564.

Canzoneri, Matthew, y Henderson, Dale (1991), Monetary policy in interdependent economies: a game-theoretic approach, Cambridge, MIT press.

Carraro, Carlo, y Giavazzi, Francesco (1988), “¿Can international policy coordination really be counterproductive?", NBER Working Paper Series 2669.

Chang, Eui; Muinhos, Marcelo, y Teixeira Joanilio (2002), "Macroeconomic coordination and inflation targeting in a two-country model", Working Paper Series 50, Banco Central do Brasil.

Clarida, Richard; Galí, Jordi, y Gertler, Mark (1999), "The Science of Monetary Policy: A New Keynesian Perspectiva”, Journal of Economic Literature, 37 (4), pp. 1661-1707.

Díaz, Carmen (2005), "La política económica en una unión monetaria: ¿independencia o coordinación?”, ICE Tribuna de Economía, 0 (824), pp. 191-207.

Dixit, Avinash, y Lambertini, Luisa (1999), "Symbiosis of Monetary and Fiscal Policies in a Monetary Union", [PDF] Consultado el 24 de abril de 2016, en: http://ssrn. com/abstract $=203628$

Ellison, Martin; Sarno, Lucio, y Vilmunen, Jouko (2007), "Caution or Activism? Mone- 
tary Policy Strategies in an Open Economy", Macroeconomic Dynamics, 11 (4), pp. 519-541.

Fernández, Jorge (2002), Teoría de juegos: su aplicación en economía, México, Colmex. Frankel, Jeffrey, y Rockett, Katharine (1988), "International Macroeconomic Policy Coordination when Policymakers Disagree on the True Model", American Economic Review, 78 (3), pp. 318-340.

Freitas, Paulo, y Muinhos, Marcelo (2001), “A Simple Model for Inflation Targeting in Brazil”, Working Paper 18, Banco Central do Brasil.

Fritz, Barbara, y Metzger, Martina (eds.) (2006), New Issues in Regional Monetary Coordination: Understanding North-South and South-South Arrangements, Basingstoke, Palgrave Macmillan.

Goodhart, Charles (1994), "Game Theory for Central Bankers: A Report to the Governor of the Bank of England", Journal of Economic Literature, 32 (1), pp. 101-114.

Hamada, Koichi (1976), “A Strategic Analysis of Monetary Interdependence”, Journal of Political Economy, 84 (4), pp. 677-700.

Jensen, Henrik (1997), "Monetary Policy Cooperation May Not Be Counterproductive", The Scandinavian. Journal of Economics, 99 (1), pp. 73-80.

Kempf, Hubert, y Thadden, Leopold (2008), "On policy interactions among nations: when do cooperation and commitment matter", Working Paper 196, Banque de France.

Krolzig, Hans (1997), "International monetary policy coordination and credibility under incomplete information", Applied Economics Discussion Paper 193, Institute of Economics and Statistics, University of Oxford

Leitemo, Kai (2004), "A game between the fiscal and the monetary authorities under inflation targeting", European Journal of PoliticalEconomy, 20 (3), pp. 709-724.

Leitemo, Kai; Roisland, Oisten, y Torvik, Ragnar (2002), "Time inconsistency and the exchange rate channel of monetary policy", The Scandinavian Journal of Economics, 104 (3), pp. 391-397.

_ (2005), "Monetary policy rules and the exchange rate channel", Applied Financial Economics, 15 (16), pp. 1165-1170.

Osborne, Martin, y Rubinstein, Ariel (1994), A course in game theory, Cambridge, The MIT Press.

Petit, Maria Luisa (1989), "Fiscal and Monetary Policy Co-Ordination: A Differential Game Approach", Journal of Applied Econometrics, 4 (2), pp. 161-179.

Rogoff, Kenneth (1985), "Can international monetary policy cooperation be counterproductive?", Journal of International Economics, 18 (3-4), pp. 199-217. 
192 ECONOMÍA: TEORÍA Y PRÁCTICA • Nueva Época, número 44, enero-junio 2016

Svensson, Lars (1999), "Inflation Targeting: Some Extensions", The Scandinavian Journal of Economics, 101 (3), pp, 337-361.

Walsh, Carl (2003), Monetary theory and policy, 2a. ed., Cambridge, The MIT Press.

Woodford, Michael (1998), “Optimal Monetary Policy Inertia”, mimeo, Princeton University. 